\title{
Conventional Linear versus Purse-string Skin Closure after Loop Ileostomy Reversal: Comparison of Wound Infection Rates and Operative Outcomes
}

\author{
Jung Ryeol Lee, Young Wan Kim, Jong Je Sung, Ok-Pyung Song, Hyung Chul Kim, Cheol-Wan Lim, \\ Gyu-Seok Cho, Jun Chul Jung, Eung-Jin Shin \\ Department of Surgery, Soonchunhyang University Bucheon Hospital, Soonchunhyang University College of Medicine, Bucheon, Korea
}

Purpose: Wound infection after an ileostomy reversal is a common problem. To reduce wound-related complications, pursestring skin closure was introduced as an alternative to conventional linear skin closure. This study is designed to compare wound infection rates and operative outcomes between linear and purse-string skin closure after a loop ileostomy reversal. Methods: Between December 2002 and October 2010, a total of 48 consecutive patients undergoing a loop ileostomy reversal were enrolled. Outcomes were compared between linear skin closure (group $L, n=30$ ) and purse string closure (group $\mathrm{P}, \mathrm{n}=18$ ). The operative technique for linear skin closure consisted of an elliptical incision around the stoma, with mobilization, and anastomosis of the ileum. The rectus fascia was repaired with interrupted sutures. Skin closure was performed with vertical mattress interrupted sutures. Purse-string skin closure consisted of a circumstomal incision around the ileostomy using the same procedures as used for the ileum. Fascial closure was identical to linear closure, but the circumstomal skin incision was approximated using a purse-string subcuticular suture (2-0 Polysorb).

Results: Between group L and P, there were no differences of age, gender, body mass index, and American Society of Anesthesiologists (ASA) scores. Original indication for ileostomy was 23 cases of malignancy (76.7\%) in group L, and 13 cases of malignancy (77.2\%) in group P. The median time duration from ileostomy to reversal was 4.0 months (range, 0.6 to 55.7 months) in group L and 4.1 months (range, 2.2 to 43.9 months) in group P. The median operative time was 103 minutes (range, 45 to 260 minutes) in group L and 100 minutes (range, 30 to 185 minutes) in group P. The median hospital stay was 11 days (range, 5 to 4 days) in group $\mathrm{L}$ and 7 days (range, 4 to 14 days) in group $\mathrm{P}(\mathrm{P}<0.001)$. Wound infection was found in 5 cases $(16.7 \%)$ in group $\mathrm{L}$ and in one case $(5.6 \%)$ in group $\mathrm{L}(\mathrm{P}=0.26)$.

Conclusion: Based on this study, purse-string skin closure after a loop ileostomy reversal showed comparable outcomes, in terms of wound infection rates, to those of linear skin closure. Thus, purse-string skin closure could be a good alternative to the conventional linear closure.

Keywords: Ileostomy reversal; Wound infection; Linear closure; Purse-string closure

\section{INTRODUCTION}

Recently, as surgical treatment for low rectal cancer, sphincter-pre-

Received: November 18, 2010 Accepted: January 30, 2011

Correspondence to: Eung-Jin Shin, M.D.

Department of Surgery, Soonchunhyang University Bucheon Hospital,

1174 Jung 1-dong, Wonmi-gu, Bucheon 420-767, Korea

Tel: +82-32-621-5249, Fax: +82-32-621-5018

E-mail: colon@schmc.ac.kr

(C) 2011 The Korean Society of Coloproctology

This is an open-access article distributed under the terms of the Creative Commons Attribution NonCommercial License (http://creativecommons.org/licenses/by-nc/3.0) which permits unrestricted noncommercial use, distribution, and reproduction in any medium, provided the original work is properly cited. serving procedures have been increasing gradually. In addition, as surgical treatments for ulcerative colitis, a total a proctocolectomy and an ileal pouch anal anastomosis are increasingly chosen. Nevertheless, it has been reported that the lower the anastomotic site becomes, the higher the risk of anastomotic leakage after a colocolostomy or a coloanal anastomosis becomes. Therefore a temporary diverting stoma for the purpose of fecal diversion to protect the anastomotic site after a proctocolectomy is increasingly used [1-3]. Commonly applied temporary diverting stomas are the ileostomy and the colostomy. In several reports, the advantages of an ileostomy are that the fistula size of an ileostomy is small, fecal odor is less, the risk of herniation is low, and complications during ileostomy creation and its reversal are fewer in comparison with 
a colostomy, and it is technically easy; thus, it is presently being widely performed [4-6].

Wound infection after an ileostomy reversal is a relatively common complication. The incidence of wound infection ranges from $0 \%$ to as high as $41 \%$, depending on investigators $[7,8]$. The causes of wound infection are a contaminated wound due to bacteria on the skin in the vicinity of the ileostomy in contact with bowel contents for a long time and the leakage of the ileostomy contents [7]. Therefore, to reduce wound infection, Banerjee [9] reported that after an ileostomy reversal, if wounds are closed by purse-string skin closure, wound infection is less, and scars become smaller, making it cosmetically superior. Sutton et al. [10] reported that in 51 patients, on whom purse-string skin closure had been performed on wounds and who had been followed up for 6 weeks, none of cases developed wound infection. Recently, Reid et al. [11] reported that a randomized prospective study was conducted on 61 patients who underwent an ileostomy reversal by dividing them into a group in which wound closure had been performed by conventional linear skin closure $(\mathrm{n}=31)$ and a group in which purse-string skin closure had been performed $(\mathrm{n}=30)$. In the purse-string skin closure group, wound infection was $6.7 \%$ ( 2 cases), which was significantly lower in comparison with the linear skin closure group (38.7\%, 12 cases).

Until now, studies analyzing the effectiveness of purse-string skin closure are rarely conducted. In this study, in order to assess the efficacy of purse-string skin closure in an ileostomy reversal, we performed wound closure by using either conventional linear skin closure or purse-string skin closure, and we compared the rates of wound infection.

\section{METHODS}

A retrospective study was performed on 48 patients who underwent a loop ileostomy reversal from December 1, 2002, to Octo- ber 15, 2010, at Soonchunhyang University Bucheon Hospital. The rates of wound infection and the early clinical outcomes of the group in which wound closure had been performed by using linear skin closure at the time of the ileostomy reversal (L group, $\mathrm{n}=$ 30 ) and of the group in which purse-string skin closure had been performed ( $\mathrm{P}$ group, $\mathrm{n}=18$ ) were compared.

At the time of wound closure, as the linear skin closure technique, first, an elliptical incision was made around the ileostomy, and after adhesiolysis of the ileostomy, with the discretion of the surgeon, a simple closure, resection and hand-sewn end-to-end anastomosis, or a resection and stapled side-to-side anastomosis was performed. Afterward, a layer-to-layer linear suturing was performed on the fascia of the rectus abdominis muscle, subcutaneous tissues, and the skin. As the purse-string skin closure technique, a circumstomal incision was made in the vicinity of the ileostomy, and adhesiolysis and anastomosis were performed identically. The fascia of the rectus abdominis muscle was sutured linearly. Subcutaneous tissues were not sutured. In regard to the skin, purse-string suturing was performed on the dermal layer with absorbable sutures (2-0 Polysorb, Covidien, MA, USA) (Fig. 1).

On the eve of surgery, the patients took two liters of laxatives (polyethylene glycol solution) with water. Prior to surgery, as prophylactic antibiotics, until the year 2008, with the discretion of the surgeon, antibiotics were administered from immediately prior to the initiation of surgery to three days to five days after surgery. After the year 2008, second generation cephalosporins were administered prior to surgery and for up to one day after surgery. Postoperative diets were initiated after confirming intestinal movement of patients who did not have gastrointestinal retention symptoms, such as vomiting or discomfort in the abdominal area. After appropriate diets and achievement of pain control, the patients were discharged under physician-patient consultation. The follow-up period was until October 30, 2010. At our outpatient clinic, wound condition was assessed (Fig. 2).
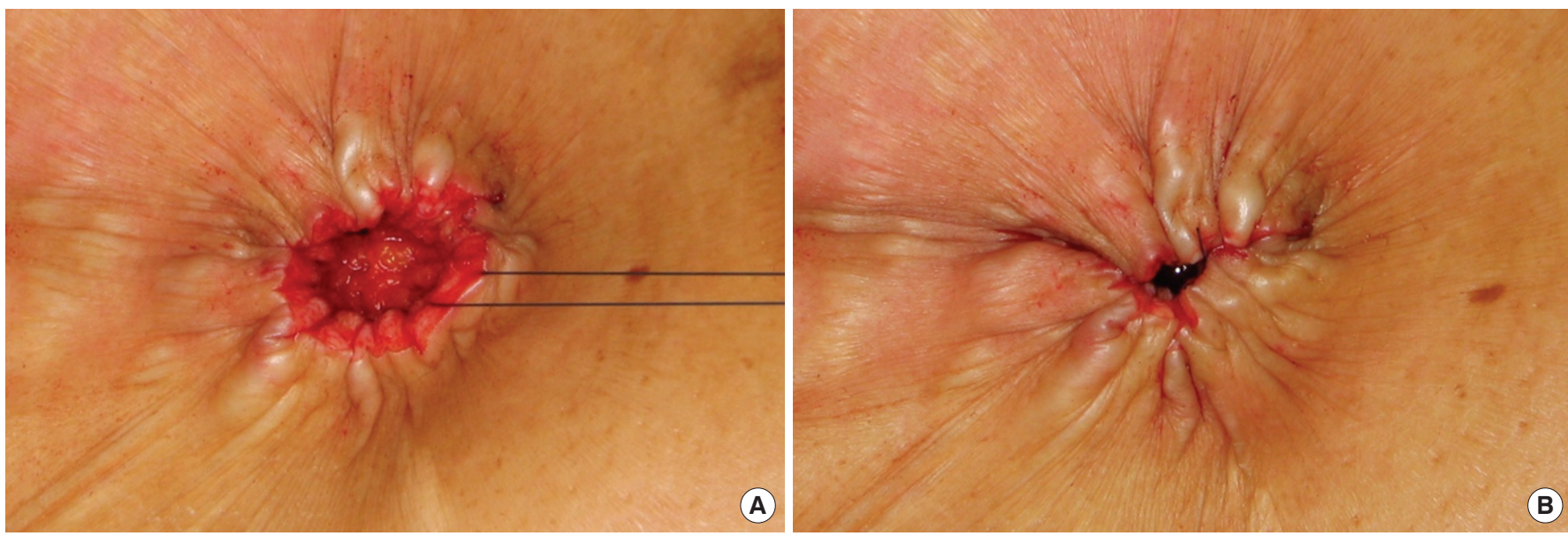

Fig. 1. (A) A subcuticlar stitch using 2-0 absorbable material was placed around the circumstomal incision in a 32-year-old male patient. (B) Tightening of the subcuticlar stitch. 

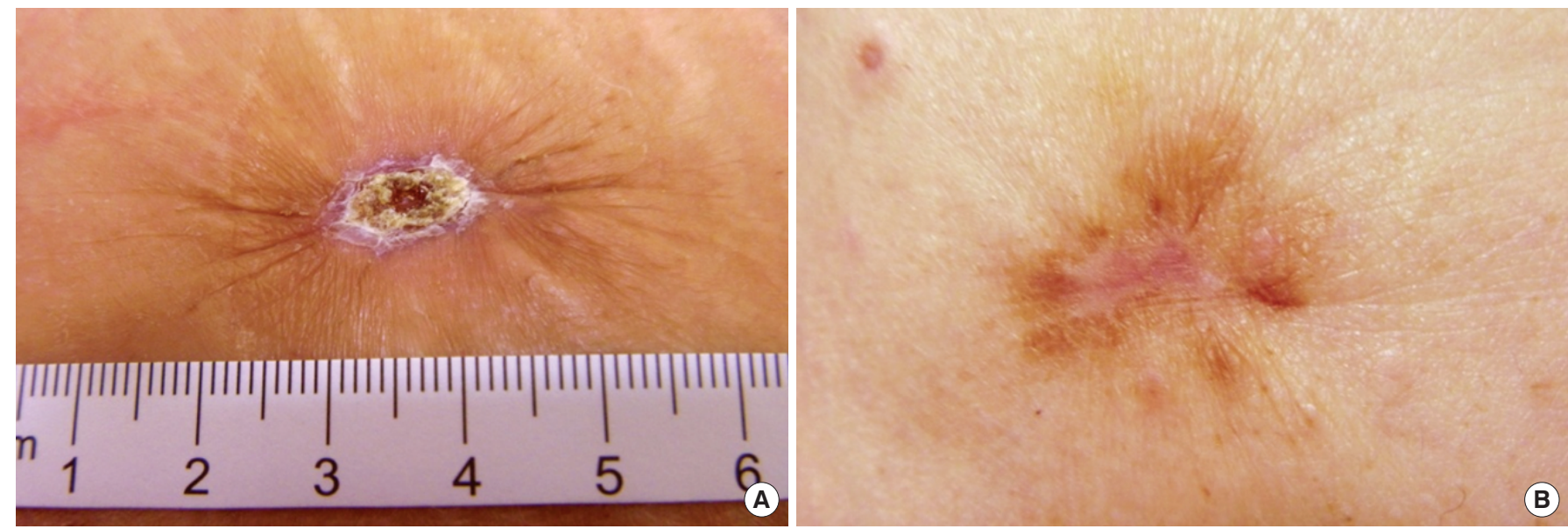

Fig. 2. (A) A small scab was seen on the wound on postoperative day 23 in a 32-year-old male patient. (B) Appearance of a scar after complete wound healing for 6 months.

Postoperative complications were defined as complications that developed within 30 days after an ileostomy reversal and that needed additional surgical or medical treatments. Postoperative wound infection was defined according to the standard of the Centers for Disease Control (CDC) as superficial or deep infection occurring in the surgical wound within 30 days after surgery [12]. Cases in which a purulent discharge was detected in the wound, cases in which bacteria were cultured, or even if bacteria were not cultured, and cases in which pain, flares, or edema was present at the wound were considered to be infected. In regard to the American Society of Anesthesiologists (ASA) score, ASA 1 was defined as normal, and ASA 2 was assigned to patients with mild systemic diseases, ASA 3 to patients with moderate systemic diseases, ASA 4 to patients with severe systemic diseases that threatened life, ASA 5 to moribund patients for whom survival would be difficult regardless of surgery, and ASA 6 to brain death patients [13].

Concerning statistical analysis, for continuous variables, the Student t-test was applied. For nominal variables, the chi-square test was applied. Findings with $P$ values lower than 0.05 were considered to be statistically significant. As a statistical analysis program, the statistical software package SPSS ver. 12.0 (SPSS Inc., Chicago, IL, USA) was used.

\section{RESULTS}

\section{The characteristics of patients}

Concerning the ratio of males to females in the subject patient group, in the L group, it was 17:13, in the P group, it was 12:6, and this difference was not statistically significant (0.49). The median age of the $\mathrm{L}$ group was 58 years, and that of the $\mathrm{P}$ group was 54 years $(\mathrm{P}=0.74)$. There were no statistically significant differences in the body mass indices and the American Society of Anesthesiologists (ASA) scores between the two groups. The underlying disease for the ileostomy was malignant tumors in 23 patients $(76.7 \%)$
Table 1. Patient characteristics

\begin{tabular}{lccc}
\hline & $\begin{array}{c}\text { Linear closure } \\
(\mathrm{n}=30)\end{array}$ & $\begin{array}{c}\text { Purse-string } \\
\text { closure }(\mathrm{n}=18)\end{array}$ & P-value \\
\hline Gender & & & 0.49 \\
$\quad$ Male & $17(56.7)$ & $12(66.7)$ & \\
$\quad$ Female & $13(43.3)$ & $6(33.3)$ & \\
Age (yr) & $12(24-82)$ & $54(27-80)$ & 0.74 \\
BMI (kg/m $\left.{ }^{2}\right)$ & $21.0(12.8-28.7)$ & $22.7(14.9-26.8)$ & 0.26 \\
ASA score & & & 0.22 \\
1 & $18(60.0)$ & $14(80.0)$ & \\
2 & $8(26.7)$ & $4(20.0)$ & \\
3 & $4(13.3)$ & $0(0.00)$ & 0.73 \\
Indication for ileostomy & & & \\
Malignancy & $23(76.7)$ & $13(77.2)$ & \\
\hline Benignity & $7(23.3)$ & $5(27.8)$ & \\
Time duration from ileostomy & $4.0(0.6-55.7)$ & $(2.2-43.9)$ & 0.87 \\
to closure (mo) & & & \\
\hline
\end{tabular}

Values are presented as number (\%) or median (range).

BMI, body mass index; ASA, American Society of Anesthesiologists.

in the $\mathrm{L}$ group and benign diseases in 7 patients (23.3\%). In the $\mathrm{P}$ group, it was malignant tumors in 13 patients $(77.2 \%)$ and malignant diseases in 5 patients $(27.8 \%)(\mathrm{P}=0.73)$. The time from ileostomy creation to its reversal in the $\mathrm{L}$ group was 4 months, and in the $\mathrm{P}$ group, it was 4.1 months $(\mathrm{P}=0.87)$ (Table 1$)$.

\section{Operative outcomes}

The median operative time of the L group was 103 minutes (range, 45 to 260 minutes), and that of the P group was 100 minutes (range, 30 to 185 minutes), the difference not being statistically significant. In the $\mathrm{L}$ group, the patient whose operative time was long (260 minutes) was a 56-year-old male patient, and 3.7 months after the 
Table 2. Operative outcomes

\begin{tabular}{lccc}
\hline & $\begin{array}{c}\text { Linear closure } \\
(\mathrm{n}=30)\end{array}$ & $\begin{array}{c}\text { Purse-string } \\
\text { closure }(\mathrm{n}=18)\end{array}$ & P-value \\
\hline Operative time (min) & $103(45-260)$ & $100(30-185)$ & 0.54 \\
Complications & & & 0.13 \\
$\quad$ Wound infection & $5(16.7)$ & $1(5.6)$ & \\
Small bowel obstruction & $3(10.0)$ & $1(5.6)$ & \\
Anastomosis leakage & $1(3.3)$ & $0(0.0)$ & \\
Hospital stay (day) & $11(5-44)$ & $7(4-14)$ & 0.00 \\
Follow-up duration (mo) & $23.3(0.2-85.9)$ & $3.7(0.1-13.4)$ & 0.00 \\
\hline
\end{tabular}

Values are presented as number (\%) or median (range).

low anterior resection and ileostomy for rectal cancer, an ileostomy reversal was performed. Because his body mass index was high $\left(27.9 \mathrm{~kg} / \mathrm{m}^{2}\right)$ and adhesion was severe, the operation time became long. At the time of an ilestomy reversal, as postoperative complications, in the L group, wound infection was seen in 5 cases (16.7\%), small bowel obstruction in 3 cases (10\%), and anastomotic leakage in 1 case (3.3\%). In the P group, wound infection was seen in 1 case (5.6\%), and small bowel obstruction in 1 case (5.6\%). The overall incidences of complications of the two groups were not statistically different $(\mathrm{P}=0.13)$.

The median hospitalization period of the $\mathrm{L}$ group was 11 days (range, 5 to 44 days), and that of the $\mathrm{P}$ group was 7 days (range, 4 to 14 days), which was significantly shorter $(P=0.00)$ (Table 2). The patient in the L group with a long hospitalization period (44 days) was a 69-year-old male patient, and 3 years prior to the diagnosis of rectal cancer, he had received a right hemicolectomy for right colon cancer. For rectal cancer, a low anterior resection and ileostomy were performed, and after 5.8 months, the ileostomy reversal was performed. The adhesion was severe because of the previous operation. Five days after surgery, a small bowel obstruction was observed; thus, nasogastric tube insertion with conservative treatments was performed. Twelve days after surgery, the nasogastric tube was removed. On day 34 , a soft diet was initiated. On day 44 , the patient was discharged.

\section{Risk factors of wound infection}

In the analysis of risk factors for wound infection, the wound closure technique, gender, age, body mass index, diabetes, hypertension, operation time, the underlying disease for the ileostomy, the time required until ileostomy reversal, the ileal anastomosis technique, and smoking were found not to be significantly associated with wound infection. Nonetheless, in cases with ASA 3, the rate of wound infection was higher than it was in cases with ASA scores 1 and $2(33.3 \%$ vs. $4.8 \% ; \mathrm{P}=0.02)$ (Table 3$)$.

\section{DISCUSSION}

This study was a retrospective analysis that compared linear skin
Table 3. Factors related to wound infection

\begin{tabular}{|c|c|c|c|}
\hline & \multicolumn{2}{|c|}{ Wound infection } & \multirow{2}{*}{ P-value } \\
\hline & Yes & No & \\
\hline Wound closure & & & 0.26 \\
\hline Linear & 25 (59.5) & $5(83.3)$ & \\
\hline Purse-string & $17(40.5)$ & $1(16.7)$ & \\
\hline Gender & & & 0.15 \\
\hline Male & 27 (64.3) & 2 (33.3) & \\
\hline Female & $15(35.7)$ & $4(66.7)$ & \\
\hline Age (yr) & & & 0.37 \\
\hline$<70$ & 37 (88.1) & $6(100.0)$ & \\
\hline$\geq 70$ & $5(11.9)$ & $0(0.0)$ & \\
\hline BMI $\left(\mathrm{kg} / \mathrm{m}^{2}\right)$ & & & 0.32 \\
\hline$<25$ & $36(85.7)$ & $6(100.0)$ & \\
\hline$\geq 25$ & $6(14.3)$ & $0(0.0)$ & \\
\hline ASA score & & & 0.02 \\
\hline 1 and 2 & 40 (95.2) & $4(66.7)$ & \\
\hline 3 & $2(4.8)$ & $2(33.3)$ & \\
\hline Diabetes & $3(7.1)$ & $1(16.7)$ & 0.43 \\
\hline Hypertension & $10(23.8)$ & $1(16.7)$ & 0.70 \\
\hline Operative time (min) & & & 0.74 \\
\hline$<100$ & $18(42.9)$ & $3(50.0)$ & \\
\hline$\geq 100$ & $24(57.1)$ & $3(50.0)$ & \\
\hline Indication for ileostomy & & & 0.61 \\
\hline Malignancy & 32 (76.2) & $4(66.7)$ & \\
\hline Benignity & $10(23.8)$ & $2(33.3)$ & \\
\hline $\begin{array}{l}\text { Time duration from } \\
\text { ileostomy to closure (mo) }\end{array}$ & & & 0.13 \\
\hline$<3$ & $9(21.4)$ & $3(50.0)$ & \\
\hline$\geq 3$ & $33(78.6)$ & $3(50.0)$ & \\
\hline Anastomosis & & & 0.69 \\
\hline Simple closure & $33(78.6)$ & $4(66.7)$ & \\
\hline $\begin{array}{l}\text { Resection \& hand-sewn } \\
\text { method }\end{array}$ & $8(19.0)$ & 2 (33.3) & \\
\hline Stapled method & $1(2.4)$ & $0(0.0)$ & \\
\hline Smoking & $18(42.9)$ & 2 (33.3) & 0.66 \\
\hline
\end{tabular}

Values are presented as number (\%).

BMI, body mass index; ASA, American Society of Anesthesiologists.

closure with purse-string skin closure for ileostomy reversal. Different from previous studies, the incidence of wound infection of the purse-string skin closure group (1 case, 5.6\%) was lower than the linear skin closure group (5 cases, $16.7 \%$ ), but this difference was not statistically significant. This is thought to be due to the small number of subject patients.

Purse-string skin closure after an ileostomy reversal is a second- 
ary closure. The advantages are that until granulation tissues grow and the skin is epithelialized, small skin defect areas become natural drainage pathways, thus avoiding wound infection. Banerjee [9] reported that the wound was completely healed in more than $90 \%$ of the patients within 8 weeks. Similarly, Sutton et al. [10] reported that after an ileostomy reversal, without wound infection, complete epithelialization was achieved within 2-4 weeks.

A shortcoming of purse-string skin closure is that because it requires a crescent-shaped skin incision, it may be difficult to obtain a surgical view for suturing the fascia of the rectus abdominis muscle in obese patients with abundant subcutaneous fat. In addition, Williams et al. [14] reported that they worried about the longer wound healing period and the increased medical cost in cases involving purse-string suture as discharge was drained continuously through the skin. Nevertheless, Reid et al. [11] reported that the wound healing period of linear skin closure was 24.6 days and that of purse-string skin closure was 20.6 days, but the difference was not statistically significant.

In regard to risk factors for wound infection after an ileostomy reversal, although the results vary depending on the investigator, Mileski et al. [15] reported that in 93 patients who received an ileostomy as well as colostomy reversal, hypertension and smoking were observed to be risk factors for wound infection. Akiyoshi et al. [16] reported that in 125 patients on whom an ileostomy had been performed after surgery for rectal cancer, being male and having wound infection at the time of rectal cancer surgery were significant risk factors for wound infection after the ileostomy reversal. According to the National Nosocomial Infections Surveillance System (NNIS), risk factors for wound infection after surgery are a contaminated or dirty wound, an ASA score higher than 3, and operative times in the 75 percentile or longer in comparison with previously known average operative times [17]. In our study, similarly, the association of wound closure technique (purse-string skin closure or linear skin closure) with the rate of wound infection was shown to be low, and in cases with ASA scores higher than 3 , wound infection was significantly higher. Therefore, for patients whose preoperative ASA score is higher than 3, more attention should be paid to preventing wound infection.

On the other hand, when purse-string skin closure is performed, the long scars that are formed at the time of conventional linear skin closure are avoided. However, the skin is creased radially, so scar cosmesis after wound healing is of concern. Reid et al. [11] objectively compared the cosmetic satisfaction level of patients about scars from linear skin closure and purse-string skin closure after an ileostomy reversal by applying the visual analog scale (1-10); the linear skin closure group was 7.3, and the purse-string skin closure group was 7.8, but the difference was not statistically significant. In our study, an objective cosmetic scale could not be used; nonetheless, in the purse-string skin closure cases, after the epithelialization process that occurred within 2-4 weeks, scabs were formed on the skin, and the scars that finally remained on the skin were circular shaped, with a diameter smaller than $2 \mathrm{~cm}$.
Several studies have reported that the hospitalization period after an ileostomy reversal did not differ according to the wound closure technique $[6,7,11]$. In our study, the median value of the hospitalization period of the purse-string skin closure group was significantly shorter; nonetheless, it is difficult to consider it as being superior due to the difference in the wound closure techniques. However, most linear closures were performed prior to 2008 while most purse-string closures were performed after 2008; thus, the selection bias of patients is thought to have exerted an influence on the hospitalization period. In addition, the trend in patient treatment has recently been changing in the direction of shorter hospital stay through the use of early recovery programs (Fast track), and this may also have had an effect on shortening the hospitalization period.

The techniques of ileostomy creation and its reversal are relatively easy; nonetheless, the incidence of complications pertinent to surgery is not low, thus requiring attention. Reviewing Korean studies pertinent to complications that developed after an ileostomy reversal, Song et al. [18] reported that after an ileostomy reversal, among 55 patients, 18 (32.7\%) developed complications; wound infection (24.3\%), small bowel obstruction (16.4\%), incisional hernia (7.9\%), etc. also developed. Kim et al. [19] reported that after an ileostomy reversal, among 164 patients, 33 (19.7\%) developed complications; small bowel obstruction (11.5\%), enteroplagia (4.2\%), incisional hernia (1.8\%), and wound infection (1.2\%) also developed. Reviewing articles in other countries, Kaidar-Person et al. [20] reviewed 26 reports and found that the incidence of overall complications after an ileostomy reversal was 2-33.1\%. Among them, small bowel obstruction was $0-15 \%$, wound infection was $0-18.3 \%$, anastomotic leakage was $0-8 \%$, and open abdominal reoperation was required in $0-12.8 \%$ of the cases. Chow et al. [21] reported that in 48 reports that analyzed 6,107 who had received an ileostomy reversal, after an ileostomy reversal, the incidence of overall complications was $17.3 \%$, the mortality rate was $0.4 \%$, and open abdominal reoperation was required in $3.7 \%$ of the cases. As complications, small bowel obstruction (7.2\%) and wound infection (5.0\%) were most prevalent, and it was pointed out that the incidence of postoperative complications was underestimated. In our results, similar to previous reports in Korea and other countries, the incidence of overall complications was $22.9 \%$ (11/48 cases), and wound infection and small bowel obstruction developed frequently.

The limitations of our study are that it was a retrospective study and, thus, was different from prospective studies, wound infection could not be defined as the primary endpoint, several variables could not be controlled in advance, and the outcomes could not be objectively compared. In addition, the time required for wound healing and the cosmetic satisfaction level of patients about surgical scars, which are important discussion points for purse-string skin closure, could not be assessed accurately. The purse-string skin closure subject group was 18 patients, which is relatively small. In addition, for the selection of patients, purse-string skin closure was introduced and performed for ileostomy reversal 
from the year 2008, thus making it difficult to compare linear skin closure with purse-string skin closure performed during the same period conclusion ileostomy reversal, wound closure by pursestring skin closure showed a wound infection rate comparable to that of conventional linear skin closure. Therefore, if cosmetic effects and so on are considered, our results suggest that the pursestring skin closure is a new alternative for wound closure.

\section{CONFLICT OF INTEREST}

No potential conflict of interest relevant to this article was reported.

\section{REFERENCES}

1. Vignali A, Fazio VW, Lavery IC, Milsom JW, Church JM, Hull TL, et al. Factors associated with the occurrence of leaks in stapled rectal anastomoses: a review of 1,014 patients. J Am Coll Surg 1997; 185:105-13.

2. Matthiessen P, Hallbook O, Rutegard J, Simert G, Sjodahl R. Defunctioning stoma reduces symptomatic anastomotic leakage after low anterior resection of the rectum for cancer: a randomized multicenter trial. Ann Surg 2007;246:207-14.

3. Law WI, Chu KW, Ho JW, Chan CW. Risk factors for anastomotic leakage after low anterior resection with total mesorectal excision. Am J Surg 2000;179:92-6.

4. Edwards DP, Leppington-Clarke A, Sexton R, Heald RJ, Moran BJ. Stoma-related complications are more frequent after transverse colostomy than loop ileostomy: a prospective randomized clinical trial. Br J Surg 2001;88:360-3.

5. Rullier E, Le Toux N, Laurent C, Garrelon JL, Parneix M, Saric J. Loop ileostomy versus loop colostomy for defunctioning low anastomoses during rectal cancer surgery. World J Surg 2001;25:274-7.

6. Rondelli F, Reboldi P, Rulli A, Barberini F, Guerrisi A, Izzo L, et al. Loop ileostomy versus loop colostomy for fecal diversion after colorectal or coloanal anastomosis: a meta-analysis. Int J Colorectal Dis 2009;24:479-88.

7. Hackam DJ, Rotstein OD. Stoma closure and wound infection: an evaluation of risk factors. Can J Surg 1995;38:144-8.

8. Garcia-Botello SA, Garcia-Armengol J, Garcia-Granero E, Espi A, Juan C, Lopez-Mozos F, et al. A prospective audit of the complications of loop ileostomy construction and takedown. Dig Surg 2004;21:440-6.

9. Banerjee A. Pursestring skin closure after stoma reversal. Dis Co- lon Rectum 1997;40:993-4.

10. Sutton CD, Williams N, Marshall LJ, Lloyd G, Thomas WM. A technique for wound closure that minimizes sepsis after stoma closure. ANZ J Surg 2002;72:766-7.

11. Reid K, Pockney P, Pollitt T, Draganic B, Smith SR. Randomized clinical trial of short-term outcomes following purse-string versus conventional closure of ileostomy wounds. Br J Surg 2010;97: 1511-7.

12. Horan TC, Gaynes RP, Martone WJ, Jarvis WR, Emori TG. CDC definitions of nosocomial surgical site infections, 1992: a modification of $\mathrm{CDC}$ definitions of surgical wound infections. Infect Control Hosp Epidemiol 1992;13:606-8.

13. American Society of Anesthesiologists. ASA physical status classification system [Internet]. Park Ridge, IL: American Society of Anesthesiologists; c1995-2011 [cited on 2010 Oct 15]. Available from: http://www.asahq.org/clinical/physicalstatus.htm.

14. Williams LA, Sagar PM, Finan PJ, Burke D. The outcome of loop ileostomy closure: a prospective study. Colorectal Dis 2008;10: 460-4.

15. Mileski WJ, Rege RV, Joehl RJ, Nahrwold DL. Rates of morbidity and mortality after closure of loop and end colostomy. Surg Gynecol Obstet 1990;171:17-21.

16. Akiyoshi T, Fujimoto Y, Konishi T, Kuroyanagi H, Ueno M, Oya $\mathrm{M}$, et al. Complications of loop ileostomy closure in patients with rectal tumor. World J Surg 2010;34:1937-42.

17. Mangram AJ, Horan TC, Pearson ML, Silver LC, Jarvis WR. Guideline for prevention of surgical site infection, 1999: Hospital Infection Control Practices Advisory Committee. Infect Control Hosp Epidemiol 1999;20:250-78.

18. Song GW, Yu CS, Lee HO, Kim MS, Namgung H, Lee GH, et al. Ileostomy related complications. J Korean Soc Coloproctol 2003; 19:82-9.

19. Kim JY, Kim JS, Hur H, Min BS, Kim NK, Sohn SK, et al. Complication and relevant factors after an ileostomy for fecal diversion in a patient with rectal cancer. J Korean Soc Coloproctol 2009;25: 81-7.

20. Kaidar-Person O, Person B, Wexner SD. Complications of construction and closure of temporary loop ileostomy. J Am Coll Surg 2005; 201:759-73.

21. Chow A, Tilney HS, Paraskeva P, Jeyarajah S, Zacharakis E, Purkayastha $S$. The morbidity surrounding reversal of defunctioning ileostomies: a systematic review of 48 studies including 6,107 cases. Int J Colorectal Dis 2009;24:711-23. 